\title{
Introduction to Citizen Journalism and Social Media Minitrack
}

\author{
Bahareh Rahmanzadeh Heravi \\ School of Information \& Communication Studies \\ University College Dublin \\ Dublin, Ireland \\ Bahareh.Heravi@ucd.ie
}

\author{
Natalie Harrower \\ Digital Repository of Ireland \\ Royal Irish Academy \\ Dublin, Ireland \\ n.harrower@ria.ie
}

The exponential growth of social media as a central communication practice, and its agility in announcing breaking news events more rapidly than traditional media, has changed the journalistic landscape: social media has been adopted as a significant source by professional journalists, and conversely, citizens are able to use social media as a form of direct reportage. Social media content now forms a significant part of the digital content generated every day, and provides a platform for voices that would not reach the broader public through traditional journalistic media alone.

Journalists and news organisations now monitor social media for breaking news and content, they use it to find sources and eyewitnesses, and to crowdsource varied perspectives on newsworthy events. They also use social media for promoting their content, attracting audiences, and driving traffic to their websites. The wealth of information social media provides is unprecedented in terms of velocity, if arguably not in terms of quality.

In this emerging environment, citizen microblogs and other user-generated content constitute an important part of history and popular memory, in particular when attempting to capture significant events and the varied perspectives that accompany these events.

This new landscape calls for technologies and methodologies to rapidly and efficiently capture, filter, verify and preserve content in a way that generates immediate value for journalistic purposes.

A host of research questions arise around the role of social media in newsrooms and in the news production lifecycle [1], and particularly in the area of real time event and story detection from social sources, e.g. [3]. This minitrack is aimed at facilitating a conversation on these topics at HICSS-50, with a particular focus on the intersection of social media and journalism, as a subset of Computational and Data Journalism. In this minitrack we aim to address a variety of research questions from both theoretical and pragmatic perspectives.

This year, in its second year, the minitrack presents a paper on a "Framework for Real-Time Event Detection using Multiple Social Media Sources" by Satya Katragadda, Ryan Benton and Vijay Raghavan. Extending the Event Detection at Onset (EDO) model, which detects an event within 3-8 minutes after the event is mentioned on Twitter [2], this paper presents a framework for social Event Detection, which uses Twitter and Tumblr as input. The detected events in this framework is validated using newswire data that is collected during a same time period. The results show that including multiple sources increases the number of detected events and also increase the quality of detected events.

We would like to extend our appreciation to the authors who submitted to this minitrack, as well as the reviewers who dedicate their time to furthering the research of our contributors. We are looking forward to the continued growth and evolution of this emerging interdisciplinary and exciting field of research.

\section{References}

[1] Heravi, B. R., Harrower, N., "Twitter journalism in Ireland: sourcing and trust in the age of social media", Information, Communication and Society, May 2016, pp. 1194-1213.

[2] Katragadda, S., Benton, R., Virani, S. and Raghavan, V., "Detection of event onset using twitter," in 2016 International Joint Conference o n Neural Networks, pp. 1539-1546, IEEE, 2016.

[3] Khare, P., Torres, P., Heravi, B. R., "What just happened? A Framework for Social Event Detection and Contextualisation", Citizen Journalism and Social Media Archiving minitrack, 48th Hawaii International Conference on System Sciences (HICSS 48), IEEE, Hawaii, US, Jan 2015. 